\title{
Nurses Responsibilities on Providing Enteral Nutrition to the Critically III Patient-An European Federation of Critical Care Nursing Associations Position Paper
}

\author{
Andreas SCHAEFER, BSc, APN ${ }^{a}$, Julie BENBENISHTY, RN, PhD $\odot^{b}$, Aleksandra \\ GUTYSZ-WOJNICKA, RN, CCRN, PhD॰ ${ }^{c}$, Jelena SLIJEPCEVIC, RN, BSN, MSN", Silvia SCELSI, RN, \\ $B S N, M N S \odot^{e}$, Arnaud BRUYNEEL, RN, CCRN, MSc ${ }^{f}$, and Mag Karin KLAS, RN ${ }^{g}$
}

In ICU, one of the nurse's roles is to ensure proper nutrition support to facilitate their recovery. However, few nutrition protocols for nurses are found. The European federation of Critical Care Nursing associations (EfCCNa) position statement provides ICU nurses with evidenced guidelines regarding caring for critically ill patients with enteral nutrition support.

Keywords: critical care unit; enteral nutrition; nurses; European Federation of Critical Care Nursing association

\section{BACKGROUND}

Nutritional status impacts wound healing, ventilator weaning, organ function, mobility, and mortality (Cederholm et al., 2017). Tian, Heighes, Allingstrup, and Doig (2018) preformed a metaanalysis of randomized controlled trials and demonstrated that better outcomes occurred when patients who were unable to eat received nutrition support within 24 to 48 hours of ICU admission. Therefore, it is essential that nurses understand nutrition assessment and application, monitor and manage severely ill patient's nutrition. In ICU, one of the nurse's roles is to provide proper nutrition to ensure support of severely ill patients' organ systems. In nourishing critically ill patients, nurses have an important role in identifying those at risk of inadequate feeding, malnutrition, and preventing complications of enteral nutrition (Dhaliwal, Cahill, Lemieux, \& Heyland, 2014; McClave et al., 2016).

\section{NURSES RESPONSIBILITIES ON PROVIDING ENTERAL NUTRITION — AN EFCCNA POSITION STATEMENT}

This European federation of Critical Care Nursing associations (EfCCNa) position paper provides a protocol for nurse to care critically ill patients with enteral nutrition. In this position statement, critically ill patient is defined as those with complex health situations, at high risk for insufficient nutrition, and in the need of an individual approach; enteral nutrition is defined as the application of nutritional supplements via oro/naso gastral tubes or devices placed into gastrointestinal area. Nurses must assure that patient's nutrition administration starts within $24 \mathrm{hr}-48 \mathrm{hr}$

\footnotetext{
${ }^{a}$ Critical Care Asklepios Klinik Schwalmstadt, Germany

${ }^{b}$ Trauma Coordinator Hadassah Hebrew University Medical Center Jerusalem, Israel. E-mail:julie@hadassah.org.il

${ }^{c}$ Critical Care Nurse Specialist, Adiunct Faculty of Health Sciences Collegium Medicum University of Warmia and Mazury, Olsztyn, Poland

${ }^{d}$ Department of Anesthesiology, Reanimatology and Intensive Care University Hospital Centre Zagreb, Croatia

e Director Nursing and Health Professions Department G. Gaslini Children's Hospital Genova, Italy

${ }^{f}$ Critical Care Department, Cliniques Universitaires St Luc, UCL, Brussels, Belgium

${ }^{g}$ Programme Director General Nursing \& Critical Care Nursing IMC University of Applied Sciences Krems, Austria
} 
Figure 1. Protocol of caring for critically ill patients with enteral nutrition.

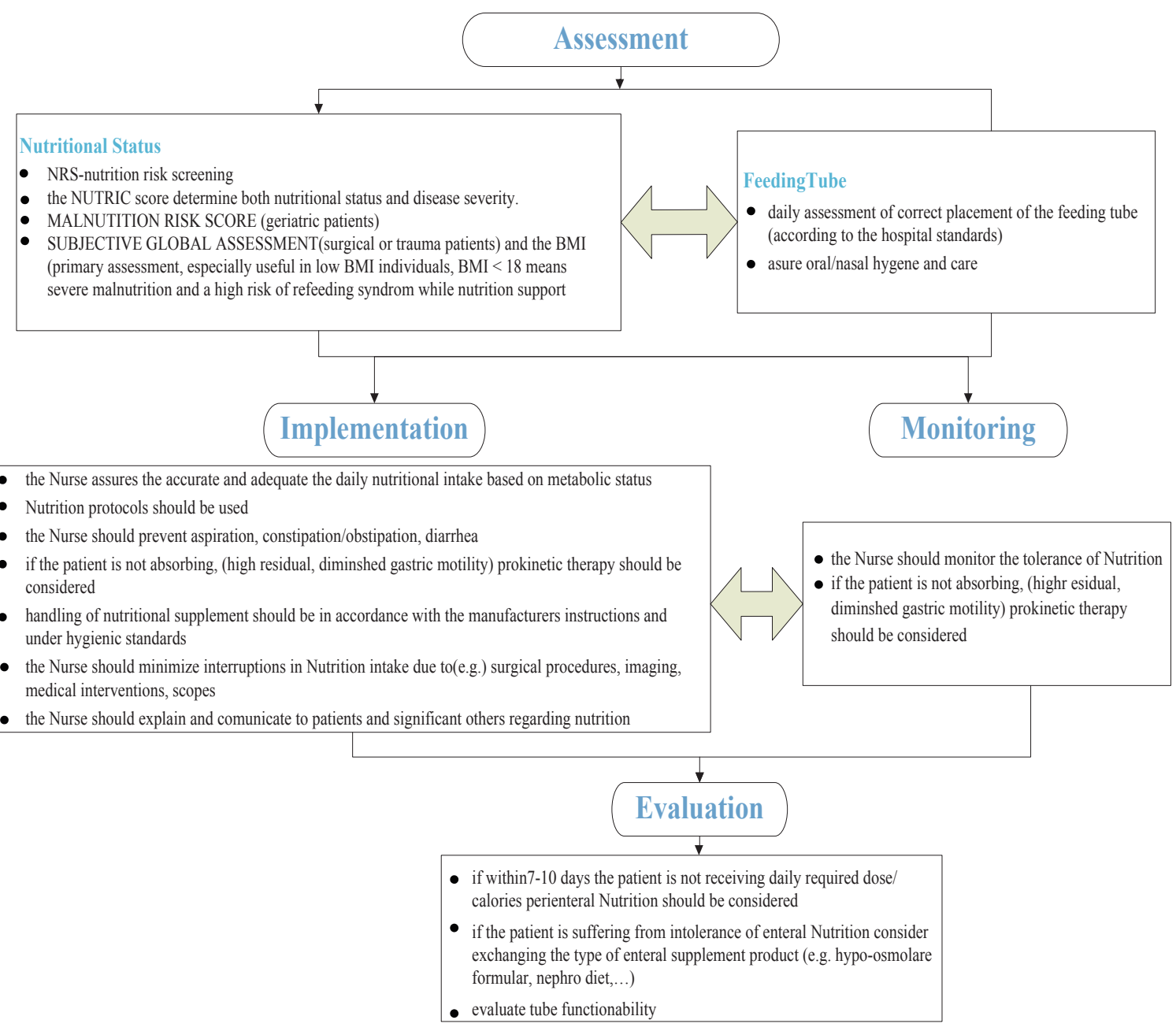

after admission to ICU (McClave et al., 2016). Then, they should assess the nutritional status and feeding tube efficacy of patients, implement and monitor enteral nutrition, and then evaluate the outcomes of enteral nutrition. Figure 1 illustrates the protocol of caring for critically ill patients with enteral nutrition.

\section{Assessment}

\section{i. Nutritional Status}

There are a number of tools that can be used for daily assessing the nutritional status, such as:

- Nutrition risk screening (NRS)

(Kondrup, Rasmussen, Hamberg, \&

Stanga, 2003)
- The Nutrition Risk in the Critically Ill (NUTRIC) score assessing both nutritional status and disease severity (Heyland, Dhaliwal, Jiang, \& Day, 2011; Kondrup, Allison, Elia, Vellas, \& Plauth, 2002)

- Malnutrition Risk Score (Ferguson, Capra, Bauer, \& Banks, 1999)

- Subjective Global Assessment (for surgical or trauma patients) and the Body Mass Index (BMI, with a $\mathrm{BMI}<18$ indicating severe malnutrition and a high risk of refeeding syndrome while nutrition support) (Cederholm et al., 2017) 
ii. Feeding Tube

- Daily assessment of the placement of the feeding tube (according to the hospital standards) to ensure its correct placement

- Assuring oral/nasal hygiene and care

2. Implementation and monitoring (enteral nutrition)

- Asurring the accurate and adequate daily nutritional intake based on metabolic status

- Nutrition protocols should be used (Kreymann, 2010; Makic, VonRueden, Rauen, \& Chadwick, 2011)

- Monitoring the tolerance of nutrition (Nguyen, 2014)

- Preventing aspiration, constipation, and diarrhoea (Blaser et al., 2017)

- If the patient is not absorbing leading to food residue and diminshed gastric motility, then prokinetic therapy should be considered (Knowles, McInnes, Elliot, Hardy, \& Middleton, 2014; Montejo et al., 2010)

- Nutritional supplement should be taken in accordance with the manufacturers instructions and under hygienic standards (National Clinical Guideline Centre, 2012)

- Minimizing interruptions in nutrition intake due to surgical procedures, imaging, medical interventions, scopes and so on

- Explaining and communicating to patients and significant others regarding nutritional support (Doenges, Moorhouse, \& Murr, 2013)

3. Evaluation

- If the patient is not receiving daily required dose/calories within 7-10 days after ICU admission, parenteral nutrition should be considered

(McClave et al., 2016)

- If the patient is suffering from intolerance of enteral nutrition, nurse can recommend considering exchanging the type of enteral supplement product (e.g., hypo-osmolare formular, nephro diet, etc.) (McClave et al., 2016; Singer et al., 2011)

- Evaluating tube functionability (Ministry of Health Singapore, 2010)

\section{CONCLUSION}

The EfCCNa position stated above provides an overview concerning the key role of nurses in enteral nutrition for critically ill patient. The position statement is underpinned with evidence-based guidelines and sources from leading experts in behalf of nourishing critically ill patients such as ESPEN (European Society of Parenteral and Enteral Nutrition), ASPEN (American Society of Parenteral and Enteral Nutrition), and ESICM (European Society of Intensive Medicine).

\section{References}

Blaser, A. R., Starkopf, J., Alhazzani, W., Berger, M. M., Casaer, M. P., Deane, A. M., \& Loudet, C. I. (2017). Early enteral nutrition in critically ill patients: ESICM clinical practice guidelines. Intensive Care Medicine, 43(3), 380398. doi:10.1007/s00134-016-4665-0

Cederholm, T., Barazzoni, R., Austin, P., Ballmer, P., Biolo, G., Bischoff, S. C., \& Jensen, G. L. (2017). ESPEN guidelines on definitions and terminology of clinical nutrition. Clinical Nutrition, 36(1), 49-64. doi:10.1016/j.clnu.2016.09.004

Dhaliwal, R., Cahill, N., Lemieux, M., \& Heyland, D. K. (2014). The Canadian critical care nutrition guidelines in 2013: An update on current recommendations and implementation strategies. Nutrition in Clinical Practice, 29(1), 2943. doi:10.1177/0884533613510948

Doenges, M. E., Moorhouse, M. F., \& Murr, A. C. (2013). Nursing diagnosis manual: Planing, individualizing, and documenting client care. Philadelphia, PA: F.A. Davis Company.

Ferguson, M., Capra, S., Bauer, J., \& Banks, M. (1999). Development of a valid and 
reliable malnutrition screening tool for adult acute hospital patients. Nutrition, 15, 458464. doi:10.1016/S0899-9007(99)00084-2

Heyland, D. K., Dhaliwal, R., Jiang, X., \& Day, A. G. (2011). Identifying critically ill patients who benefit the most from nutrition therapy: The development and initial validation of a novel risk assessment tool. Critical Care, 15(6), 268. doi:10.1186/cc10546

Knowles, S., McInnes, E., Elliot, D., Hardy, J., \& Middleton, S. (2014). Evaluation of the implementation of a bowel management protocol in intensive care: Effect on clinician practices and patient outcomes. Journal of Clinical Nursing, 23, 716-730. doi:10.1111/jocn. 12448

Kondrup, J., Allison, S. P., Elia, M., Vellas, B., \& Plauth, M. (2002). ESPEN guidelines for nutrition screening 2002. Clinical Nutrition, 22(4), 415-421. doi:10.1016/S02615614(03)00098-0

Kondrup, J., Rasmussen, H. H., Hamberg, O., \& Stanga, Z. (2003). Nutritional risk screening (NRS 2002): A new methodbased on an analysis of controlled clinical trials. Clinical Nutrition, 22(3), 321-336. doi:10.1016/S0261-5614(02)00214-5

Kreymann, G. (2010). New developments in clinical practice guidelines. South African Journal of Clinical Nutrition, 23(Suppl. 1), 29-32. doi:10.1080/16070658.2010.11734266

Makic, M. B. F., VonRueden, K. T., Rauen, C. A., \& Chadwick, J. (2011). Evidence-based practice habits: Putting more sacred cows out to pasture. Critical Care Nurse, 31(2), 38-62. doi:10.4037/ccn2011908

McClave, S. A., Taylor, B. E., Martindale, R. G., Warren, M. M., Johnson, D. R., Braunschweig, C., \& Gervasio, J. M. (2016). Guidelines for the provision and assessment of nutrition support therapy in the adult critically ill patient. Journal of Parenteral and Enteral Nutrition, 4O(2), 159211. doi:10.1177/0148607115621863
Ministry of Health Singapore. (2010). Nursing clinical practice guidelines 1/2010 nursing management of nasogastric tube feeding in adult patients. Singapore. Retrieved from https://www.moh.gov.sg/home

Montejo, J. C., Minambres, E., Bordeje, L., Mesejo, A., Acosta, J., Heras, A., \& Manzanedo, R. (2010). Gastric residual volume during enteral nutrition in ICU patients: The REGANE study. Intensive Care Medicine, 36(8), 1386-1393. doi:10.1007/s00134-0101856-y

National Clinical Guideline Centre. (2012). Infection: Prevention and control of healthcare-associated infections in primary and community care: Partial update of NICE clinical guideline 2. Royal College of Physicians (UK). Retrieved from https://www.ncbi.nlm.nih.gov/pubmed/23285 500

Nguyen, N. Q. (2014). Pharmacological therapy of feed intolerance in the critically ills. World Journal of Gastrointestinal Pharmacology and Therapeutics, 5(3), 148. doi:10.4292/wjgpt.v5.i3.148

Singer, P., Anbar, R., Cohen, J., Shalita-Chesner, M., Lev, S., Grozovski, E., \& Madar, Z. (2011). The tight calorie control study (TICACOS) a prospective, randomized, controlled pilot study of nutritional support in critically ill patients. Intensive Care Medicine, 37(4), 601609. doi:10.1007/s00134-011-2146-z

Tian, F., Heighes, P. T., Allingstrup, M. J., \& Doig, G. S. (2018). Early enteral nutrition provided within 24 hours of ICU admission: A meta-analysis of randomized controlled trials. Critical Care Medicine, 46(7), 1049-1056. doi:10.1097/CCM.0000000000003152

Disclosure. The authors have no relevant financial interest or affiliations with any commercial interests related to the subjects discussed within this article. 